\title{
Solution of three-dimensional unsteady external flow using a coupled arbitrary Lagrangian FEM-BEM model
}

\author{
D.L. Young ${ }^{\mathrm{a}, *}$, J.T. Chang ${ }^{\mathrm{a}}$, T.I. Eldho ${ }^{\mathrm{b}}$

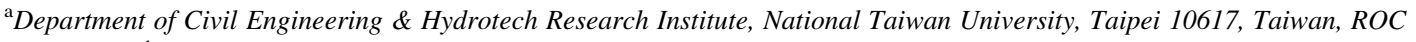 \\ ${ }^{\mathrm{b}}$ Department of Civil Engineering, Indian Institute of Technology, Bombay, Mumbai 400 076, India
}

Received 25 July 2002; revised 9 January 2003; accepted 16 August 2003

\begin{abstract}
A computational model has been developed in this paper to solve three-dimensional unsteady incompressible viscous flow problems in external flow fields. The model is based on primitive variables in Navier-Stokes equations under transient conditions. The model can be used to solve infinite boundary value problems by extracting the boundary effects on a specified finite computational domain using projection method of the Navier-Stokes equations. The momentum equation of fluid motion is solved using the three-step finite element method. The external flow field is simulated using the boundary element method by solving a pressure Poisson equation by considering the pressure to be zero at the infinite boundary. Arbitrary Lagrangian-Eulerian method is incorporated in the present model to solve the moving boundary problems. The model has been applied initially to simulate a cubic cavity flow problem for verification purpose and further used to simulate the flow past a square cylinder in two dimensions. Finally, the external flow problem of flow induced by the movement of a sphere inside a viscous flow field in three dimensions has also been considered. The simulation results are found to be reasonable and satisfactory.

(C) 2003 Elsevier Ltd. All rights reserved.
\end{abstract}

Keywords: Navier-Stokes equations; Arbitrary Lagrangian-Eulerian method; Boundary elements; Finite elements; Three-dimensional external flows

\section{Introduction}

The solutions of three-dimensional laminar viscous flows in external flow fields and related fluid structure interaction phenomena are very important in many engineering fields like wind across high-rise buildings, offshore structures, moving vehicles and aeronautics. The main difficulty in simulating these flows lies in setting up the boundary conditions of the computational domain to simulate the external flow that has infinite domain. It is also well-known that the vortex shedding around the structure plays an important role in characterizing various types of fluidinduced structural vibrations in such problems [1]. It is necessary to deal with the unsteady Navier-Stokes equations in three-dimensions in an infinite domain to develop a computational model for this class of problems.

Numerical methods like finite volumes (FVM), finite differences (FDM), finite elements (FEM) and boundary

\footnotetext{
* Corresponding author. Tel.: +886-2-2362-6114; fax: +886-2-23639258.

E-mail address: dlyoung@hy.ntu.edu.tw (D.L. Young).
}

elements (BEM) are most commonly used for the solutions of three-dimensional incompressible viscous flows. Braza et al. [2], for example, used a second-order finite volume method to solve unsteady Navier-Stokes equations and investigated the dynamical characteristics of the pressure and velocity fields of the unsteady incompressible laminar flows. The numerical simulation of unsteady incompressible Navier-Stokes equations using FDM has been reported in numerous studies (for example, [3] using vorticity-stream function formulation). In the last two decades, FEM and BEM have become the most prominent numerical methods for the solution of incompressible viscous flows. Various researchers have explored the different applications of FEM and BEM for the solution of different forms of the NavierStokes equations in two-dimensions and three-dimensions.

Various forms of finite element formulations are described in literature for the solution of incompressible viscous flows. Galerkin schemes cannot be used effectively to deal with the convective terms. Therefore, other variations of FEM like Petrov-Galerkin formulations [4] and Taylor-Galerkin schemes [5] were developed. A threestep finite element formulation for the solution of unsteady 
incompressible viscous flow has been developed by Jiang and Kawahara [6], by using the Taylor-Galerkin scheme. Different forms of BEM like direct BEM, indirect BEM and dual reciprocity BEM [7] have also been used to solve fluid dynamics problems. A coupled BEM-FEM scheme in combination with other techniques are developed in the present investigations for the solution of threedimensional incompressible viscous flow problems in external flow filed.

FEM applications to fluid structure interaction problems have first been reported by Belytschko and Kennedy [8] using a purely Lagrangian method for the kinematical description of the fluid domain. Such approach is limited due to its inability to cope easily with the strong distortions in fluid structure interaction that often characterize the flows of interest. On the other hand, the distortions can be handled easily if purely Eulerian concept is used. However, it is at the expense of precise interface definition and complexity in handling fluid structure coupling. Lagrangian method cannot satisfactorily handle the material distortions and Eulerian method has got drawback in handling convective effects. Thus, combinations of Lagrangian-Eulerian methods with different modifications are in common use [9].

Due to the limitations of purely Lagrangian and Eulerian schemes, arbitrary Lagrangian-Eulerian (ALE) techniques have been developed, first in the finite differences by Noh [10] and Hirt et al. [11], among others and in FEM by Donea et al. [12] and Hughes et al. [13]. The ALE techniques are based on the arbitrary movement of the reference frame, which is continuously rezoned in order to allow a precise description of moving interfaces.

Recently, Young et al. [14] developed a novel computational procedure for external flows by coupling BEM and ALE FEM and applied the formulation to the twodimensional laminar flows in external flow fields. Young et al. [15] used a coupled BEM and three-step FEM model for the simulation of laminar vortex shedding for flow past cylinders and also for direct numerical simulation of highReynolds flow around circular cylinders [16].

The earlier developed computational procedure in twodimensions described above is extended to solve the threedimensional incompressible viscous flow problems in external laminar flow fields in the present investigations. The model is based on the projection method of the unsteady Navier-Stokes equations in terms of pressure and velocity. The momentum equation of the flow domain is solved using a three-step explicit finite element scheme. The pressure Poisson equation for the external flow field is simulated by the BEM. Use of BEM for the solution of the pressure Poisson equation helps to handle the infinite domain of the external flow problem from a finite discrete domain efficiently, as the fundamental solutions used in the BEM formulations automatically satisfy the conditions at the infinity. The ALE scheme is employed to deal with the moving boundary, such as the motion of an impulsively moving sphere in a viscous fluid.
The feasibility and accuracy of the developed numerical model has been demonstrated using the well-known model problem of flow in a driven cubic cavity. Further, the model is applied for the simulation of two-dimensional flow past a square cylinder problem and finally, the movement of a sphere in a viscous flow field in three-dimensions.

\section{Governing equations}

The governing equations of the motion of an incompressible viscous fluid flow in three-dimensions can be expressed by the Navier-Stokes equations and continuity equation and written in Cartesian tensor notation in nondimensional form as [17]

$$
\begin{aligned}
& \frac{\partial u_{i}}{\partial x_{i}}=0 \\
& \frac{\partial u_{i}}{\partial t}+\frac{u_{j} \partial u_{i}}{\partial x_{j}}=-\frac{\partial p}{\partial x_{i}}+\frac{1}{R e}\left(\frac{\partial^{2} u_{i}}{\partial x_{j} \partial x_{j}}\right)
\end{aligned}
$$

where $u_{i}, i=1,2,3$, are the three components $(u, v$ and $w)$ of the velocity vectors in the $x$ -,$y$-, $z$-directions, respectively. Here the following dimensionless variables are used

$x=x^{*} / D, \quad y=y^{*} / D, \quad z=z^{*} / D, \quad u=u^{*} / u_{0}$,

$v=v^{*} / u_{0}, \quad w=w^{*} / u_{0}, \quad t=t^{*} u_{0} / D$,

$p=p^{*} / \rho u_{0}^{2}, \quad R e=u_{0} D / v$

The star denotes a dimensional variable, and $t$ refers to time. Further, $p$ represents pressure, $v$ represents kinematic viscosity, $\rho$ refers to mass density, $R e$ is the Reynolds number, $D$ is a characteristic dimension used to normalize the variables and $u_{0}$ is the uniform velocity of the flow field.

Appropriate initial and boundary conditions should be prescribed for the solution of external transient problems. Some known values of velocities can be prescribed as the initial conditions given by

$u=\bar{u}_{0} ; \quad v=\bar{v}_{0} ; \quad w=\bar{w}_{0}$

At the inlet plane of the finite flow domain, a uniform flow profile can be prescribed

$u=U ; \quad v=0 ; \quad w=0$

On the other boundaries of the finite domain, prescribed velocities or flow gradient and the non-slip boundary conditions can be assumed. Such conditions, however, are not essential in the present model.

As far as external flows are concerned, the outer boundaries are located at the infinity and the pressure at infinity is assumed as zero. Due to the limitations of computational facilities, it is assumed in numerical computations that the computational domain is limited to the finite region. A uniform flow is assumed outside the finite region such that continuity equation is valid. Further 
convective acceleration can be neglected and the pressure gradient in the flow direction is zero [18]. Hence, it can be assumed that

$\nabla^{2} p=0$

Eq. (6) is valid out of the finite computational domain, where the fluid is assumed undisturbed. Therefore, it is found to automatically satisfy the radiation boundary condition. Hence only the inflow boundary condition is needed as the boundary requirement of the computational domain. The outflow boundary conditions are not necessary to be specified in the model described, as it will be automatically satisfied by the estimated pressure gradient. A sample computational domain for the illustrated examples considered in the present investigation is shown in Fig. 1. Boundary condition of fixed body in the flow is set as no-slip boundary. Depending on the ALE description employed, the boundary of the moving body is specified as the velocity of flow equal to that of the body.

The coefficient of drag and coefficient of lift on solid body and Strouhal number $S_{t}$ are found from the following equations

$$
\begin{aligned}
& C_{\mathrm{d}}=\frac{F_{\mathrm{d}}}{1 / 2 \rho u_{0}^{2} A}, \quad C_{l}=\frac{F_{l}}{1 / 2 \rho u_{0}^{2} A}, \quad S_{t}=\frac{f D}{u_{0}} \\
& F_{\mathrm{d}}=\oint_{s} p_{s} \cos \phi \mathrm{d} A+\oint_{s} \tau_{s} \sin \phi \mathrm{d} A \\
& F_{1}=\oint_{s} p_{s} \sin \phi \mathrm{d} A+\oint_{s} \tau_{s} \cos \phi \mathrm{d} A
\end{aligned}
$$

Here $u_{0}$ is the characteristic fluid velocity, $F_{\mathrm{d}}$ is the drag force, $F_{1}$ is the lift force, $f$ is the frequency of the oscillation, $\tau_{s}$ is the shear force acting on the body, $p_{s}$ is the pressure acting on the body, $A$ is projected area, $\phi$ is the angle between the direction of flow and the normal to the surface element.

\section{Numerical formulation}

A coupled BEM-FEM approach is used to solve the governing differential equations. The moving boundaries in the problem are treated with the ALE scheme. The numerical formulation is briefly described in this section.

\subsection{Arbitrary Lagrangian-Eulerian scheme}

The ALE technique developed in FEM by Donea et al. [12] and Hughes et al. [13] describes the fundamental kinematical relations derived by defining three domains in space called, the spatial domain, the material domain and the referential domain, and mapping between the domains. When the material body includes free boundaries or is subjected to large deformation, a mixed description of partially Lagrangian and partially Eulerian (also called referential description) may be more adapted for numerical models. In ALE description using FEM, the spatial domain is the moving mesh and the referential domain is the reference states of the moving mesh.

In the ALE scheme described by Hughes et al. [13] and Huerta and Liu [9], let $B\left(X_{i}, t\right)$ represents the material

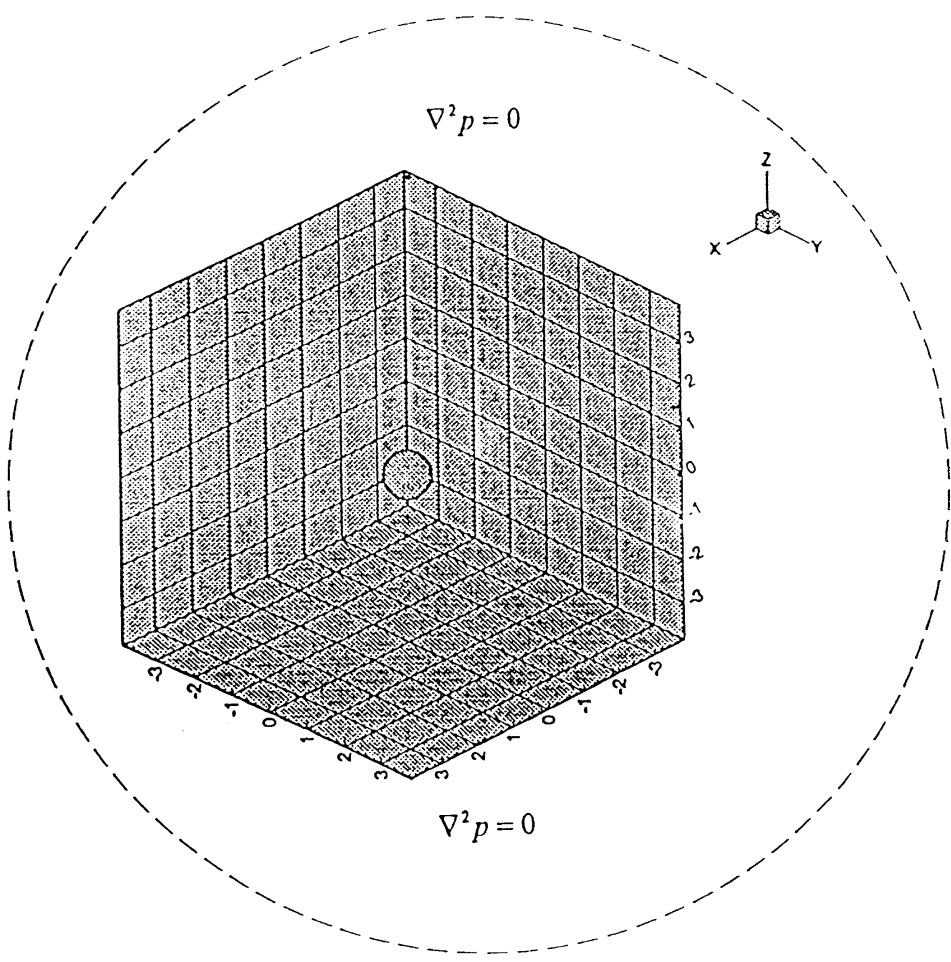

Fig. 1. Computational domain for a sample external flow problem. 
domain (Lagrangian), $B\left(x_{i}, t\right)$ represents the spatial domain (Eulerian) and $B\left(\xi_{i}, t\right)$ represents the referential domain. The Lagrangian-Eulerian mapping can be described by $x_{i}=$ $x_{i}\left(X_{i}, t\right)$ and the Eulerian-referential mapping can be described by $x_{i}=x_{i}\left(\xi_{i}, t\right)$ Considering a physical property $g\left(x_{i}, t\right)$ expressed in a spatial domain. Using the ALE scheme, the time derivative of $g$ can be written as

$$
\left.\frac{\partial g}{\partial t}\left(X_{i}, t\right)\right|_{X_{i}}=\left.\frac{\partial g}{\partial t}\left(\xi_{i}, t\right)\right|_{\xi_{i}}+c_{i} \frac{\partial g}{\partial x_{i}}\left(x_{i}, t\right)
$$

In Eq. (10), $c_{i}$ is the convective velocity described as

$c_{i}=u_{i}-\tilde{u}_{i}$

Here $u_{i}$ is the material velocity and $\tilde{u}_{i}$ is the mesh velocity. In the Eulerian description, the mesh velocity is zero, $\tilde{u}_{i}=0$, and on the other hand in the Lagrangian description, $\tilde{u}_{i}=u_{i}$.

From the above described ALE scheme, the governing Eqs. (1) and (2) can be rewritten as

$\frac{\partial u_{i}}{\partial x_{i}}=0$

$\left.\frac{\partial u_{i}}{\partial t}\right|_{\xi_{i}}+\left(u_{j}-\tilde{u}_{j}\right) \frac{\partial u_{i}}{\partial x_{j}}=-\frac{\partial p}{\partial x_{i}}+\frac{1}{R e}\left(\frac{\partial^{2} u_{i}}{\partial x_{j} x_{j}}\right)$

where $\tilde{u}_{j}$ is the mesh velocity.

While using the ALE concept in FEM, spatial domain is the moving mesh and the referential domain is the reference-state of moving mesh. On the fixed discrete domain, the mesh velocity is assumed to be zero and on the moving interface, the mesh velocity should be equal to the velocity of the moving object. A linear relationship of mesh velocity is assumed between the fixed discrete domain and moving interface [19].

\subsection{Three-step FEM formulation for Navier-Stokes equations}

The mass-momentum Navier-Stokes equations are approximated in the present study using an explicit threestep FEM based on a Taylor series expansion in time [6]. A function $f$ in time can be represented using Taylor's series as

$f(t+\Delta t)=f(t)+\Delta t \frac{\partial f(t)}{\partial t}+\frac{\Delta t^{2}}{2} \frac{\partial^{2} f(t)}{\partial t^{2}}+\frac{\Delta t^{3}}{6} \frac{\partial^{3} f(t)}{\partial t^{3}}+O\left(\Delta t^{4}\right)$

Approximating Eq. (14) up to the third-order accuracy, the following three-step formulation can be written

$$
\begin{aligned}
& f\left(t+\frac{\Delta t}{3}\right)=f(t)+\frac{\Delta t}{3} \frac{\partial f(t)}{\partial t} \\
& f\left(t+\frac{\Delta t}{2}\right)=f(t)+\frac{\Delta t}{2} \frac{\partial f(t+\Delta t / 3)}{\partial t} \\
& f(t+\Delta t)=f(t)+\Delta t \frac{\partial f(t+\Delta t / 2)}{\partial t}
\end{aligned}
$$

When Eqs. (15)-(17) are discretized using the standard Galerkin FEM in space and are solved, the resulting scheme is referred to as the three-step FEM [6]. This method has all the advantages of Taylor-Galerkin method such as thirdorder accuracy. A detailed description of the third-order accuracy of the three-step FEM has been described in Jiang and Kawahara [6].

Using a projection method of the Navier-Stokes equations [20], the present three-step FEM scheme can be derived in two phases. In the first phase, Eq. (13) is converted in the following steps

Step 1

$$
\frac{u_{i}^{n+1 / 3}-u_{i}^{n}}{\Delta t / 3}=-\left[\left(u_{j}^{n}-\tilde{u}_{j}^{n}\right) \frac{\partial u_{i}^{n}}{\partial x_{j}}\right]-\frac{\partial p^{n}}{\partial x_{i}}+\frac{1}{R e} \nabla^{2} u_{i}^{n}
$$

Step 2

$\frac{u_{i}^{n+1 / 2}-u_{i}^{n}}{\Delta t / 2}=-\left[\left(u_{j}^{n+1 / 3}-\tilde{u}_{j}^{n}\right) \frac{\partial u_{i}^{n+1 / 3}}{\partial x_{j}}\right]-\frac{\partial p^{n}}{\partial x_{i}}+\frac{1}{R e} \nabla^{2} u_{i}^{n+1 / 3}$

Step 3

$\frac{u_{i}^{*}-u_{i}^{n}}{\Delta t}=-\left[\left(u_{j}^{n+1 / 2}-\tilde{u}_{j}^{n}\right) \frac{\partial u_{i}^{n+1 / 2}}{\partial x_{j}}\right]+\frac{1}{R e} \nabla^{2} u^{n+1 / 2}$

Here $u_{i}^{*}$ is the apparent velocity. The present velocity can be derived on the other hand, as

$u_{i}^{n+1}=u_{i}^{*}-\Delta t \frac{\partial p^{n+1}}{\partial x_{i}}$

Spatial discretization of Eqs. (18)-(20), are performed by the standard Galerkin method using eight-point tri-linear elements [21]. Resulting finite element equations for various steps are as follows:

For step 1

$$
\begin{aligned}
M_{i j} \frac{\bar{u}_{j}^{n+1 / 3}-\bar{u}_{j}^{n}}{\Delta t / 3}= & -A_{i j}^{n} \bar{u}_{j}^{n}-\bar{B}_{i j} p_{j}^{n}-\frac{1}{R e} S_{i j} \bar{u}_{j}^{n} \\
& +\int_{\partial \Omega} \frac{N_{i}}{R e}\left(\frac{\partial \bar{u}^{n}}{\partial n}\right) \mathrm{d} S
\end{aligned}
$$

For step 2

$$
\begin{aligned}
M_{i j} \frac{\bar{u}_{j}^{n+(1 / 2)}-\bar{u}_{j}^{n}}{\Delta t / 2}= & -A_{i j}^{n+(1 / 3)} \bar{u}_{j}^{n+(1 / 3)}-\bar{B}_{i j} p_{j}^{n}-\frac{1}{R e} S_{i j} \bar{u}_{j}^{n+(1 / 3)} \\
& +\int_{\partial \Omega} \frac{N_{i}}{\operatorname{Re}}\left(\frac{\partial \bar{u}^{n+(1 / 3)}}{\partial n}\right) \mathrm{d} S
\end{aligned}
$$

For step 3

$$
\begin{aligned}
M_{i j} \frac{\bar{u}_{j}^{*}-\bar{u}_{j}^{n}}{\Delta t}= & -A_{i j}^{n+1 / 2} \bar{u}_{j}^{n+1 / 2}-\frac{1}{R e} S_{i j} \bar{u}_{j}^{n+1 / 2} \\
& +\int_{\partial \Omega} \frac{N_{i}}{\operatorname{Re}}\left(\frac{\partial \bar{u}^{n+1 / 2}}{\partial n}\right) \mathrm{d} S
\end{aligned}
$$


Eq. (21) can be discretized as

$M_{i j} \bar{u}_{j}^{n+1}=M_{i j} \bar{u}_{j}^{*}-\Delta t \bar{B}_{i j} p_{j}^{n+1}$

where

$M_{i j}=\int_{\Omega} N_{i} N_{j} \mathrm{~d} \Omega, \quad A_{i j}^{n}=\int_{\Omega} N_{i}\left(\bar{u}_{k}^{n} \frac{\partial N_{j}}{\partial x_{k}}\right) \mathrm{d} \Omega$

$\bar{B}_{i j}=\int_{\Omega} N_{i} \frac{\partial N_{j}}{\partial x_{k}} \mathrm{~d} \Omega, \quad S_{i j}=\int_{\Omega}\left(\frac{\partial N_{i}}{\partial x_{k}} \frac{\partial N_{j}}{\partial x_{k}}\right) \mathrm{d} \Omega$

Here $N_{i}, N_{j}$ and $N_{k}$ are the shape functions and 'bar' stands for the three components associated with $x$-, $y$ - and $z$ directions.

After assembling the system and applying the initial and boundary conditions, the system of equations is solved using the Jacobi iteration scheme. Before calculating the velocities in the current time step using Eq. (21), the pressure and its derivatives are to be found in the second phase. Combining the continuity Eq. (12) and taking the gradient of Eq. (21), the pressure Poisson equation is derived to correct the velocity equation as

$\nabla^{2} p^{n+1}=\frac{1}{\Delta t}\left(\frac{\partial u_{i}^{*}}{\partial x_{i}}\right)$

The pressure Poisson equation is solved by BEM formulation given in Section 3.3. The boundary conditions are derived by solving Eq. (6) on the infinite domain from the finite discrete domain. The boundary conditions for the solid surface are obtained from Eq. (21). The second phase of the numerical scheme, essentially implicit is obtained from the first phase and hence an iterative procedure is necessary between the solutions of $\bar{u}^{n+1}$ and $p^{n+1}$.

\subsection{BEM formulation for Poisson equation}

Consider the Poisson type pressure Eq. (26) in $p$ and $u_{i}^{*}$

$\nabla^{2} p=\frac{1}{\Delta t}\left(\frac{\partial u_{i}^{*}}{\partial x_{i}}\right)=b$

with appropriate pressure boundary conditions. An iterative scheme is used in the present model, such that the velocity $u_{i}^{*}$ is known in the current iteration and time step is estimated from the previous step by solving the NavierStokes equations.

Let $p^{*}$ be the fundamental solution of the Laplace equation in three dimensions, defined as $p^{*}=1 /(4 \pi r)$, where $r$ is the distance from the collocation point $(k)$ to other field points $(i)$ given by

$r=\sqrt{\left(x_{k}-x_{i}\right)^{2}+\left(y_{k}-y_{i}\right)^{2}+\left(z_{k}-z_{i}\right)^{2}}$

By applying the Green's second identity theorem to Eq. (27) and using the standard boundary element procedure [22], the following boundary integral equation can be obtained

$C_{i} p_{i}+\int_{\Gamma} p q^{*} \mathrm{~d} \Gamma+\int_{\Omega} b p^{*} \mathrm{~d} \Omega=\int_{\Gamma} q p^{*} \mathrm{~d} \Gamma$

Here $C_{i}$ is the Green's constant, $q=\partial p / \partial n$ and $q^{*}=\partial p^{*} / \partial n$.

Eq. (29) includes boundary integrals and domain integrals. The domain integration is performed in the present model by subdividing the domain into a series of internal cells, on each of which a numerical integration is performed. Here, bilinear elements are used for the boundary discretization and the three-dimensional isoparametric quadrilateral cells are used for the internal discretization. Details of the element properties, shape functions, coordinate transformation and numerical integration used here are described in Brebbia et al. [22].

If the domain is discretized into $M$ internal cells, the domain integral can be expressed as

$D_{i}=\int_{\Omega} b p^{*} \mathrm{~d} \Omega=\sum_{e=1}^{M}\left[\sum_{k=1}^{N I} w_{k}\left(b p^{*}\right)_{k}\right] \Omega_{e}$

where the integral has been approximated by a summation over different cells ( $e$ varies from 1 to $M$ ), $w_{k}$ are the Gauss integration weights, the function $\left(b p^{*}\right)_{k}$ needs to be evaluated at integration points $k$ on each cell ( $k$ varies from 1 to $N I$, where $N I$ is the total number of integration points on each cell) and $\Omega_{e}$ is the volume of cell $e$. The term $D_{i}$ is the result of the numerical integration and is different for each position $i$ of the boundary nodes.

Assuming that the boundary of the domain is discretized into NE linear elements with $N$ nodes, Eq. (29) can be discretized and written in matrix form

$C_{i} p_{i}+\sum_{j=1}^{N} \bar{H}_{i j} p_{j}+D_{i}=\sum_{j=1}^{N} G_{i j} q_{j}$

Combining the effect of the constant term $C$ with the $\bar{H}$ matrix

$H p+D=G q$

In Eq. (32), the boundary conditions are introduced and the known values are taken to the right hand side to form a system of linear equations, which are solved using Gauss elimination to find the boundary unknowns first, and the internal values are found from Eq. (29). To solve Eq. (6), the same formulation given above can be used without the domain integral term. Eq. (6) is solved for the infinite domain from the discrete finite domain.

The main advantage of using BEM in the solution of the pressure Poisson equation is the effectiveness of BEM to deal with infinite domain problems. Here the boundary conditions of pressure on the infinite computational domain are known which is used to solve the finite domain problem. As the fundamental solution used in the BEM formulation automatically satisfy the conditions at the infinity, use of BEM in the solution of the pressure Poisson equation and Laplace equation would be very efficient. Other numerical 
methods like FDM or FEM are not efficient as BEM, to solve the pressure Poisson equation in infinite domain from a finite discrete domain.

\section{Solution procedure}

An iterative scheme is used in the solution of the Navier-Stokes equations. The computational procedure adopted in the present model includes the following iterative steps:

For the time step $n=1$,

(1) Assume pressure $p=0$ at infinite domain, and solve the pressure Laplace equation (Eq. (6)) outside the computational domain and pressure Poisson equation (Eq. (26)) inside the computational domain and get the pressure boundary conditions on the boundaries of the computational domain. These equations are solved simultaneously using the compatibility and equilibrium conditions of continuity of pressure and flux at the common boundary [22].

(2) Solution of the Navier-Stokes equations using threestep FEM and projection method.

- Solve for the unknown apparent velocity values (Eq. (20))

- Calculate the pressure distribution for the current time step from the pressure Poisson equation (Eq. (26)) using BEM.

- Determine the new velocity values by solving Eq. (21).

(3) Check for convergence of the velocity and pressure components in the present iteration, for example

$$
\frac{\left|w_{k+1}^{n}-w_{k}^{n}\right|}{\left|w_{k}^{n}\right|} \leq 0.001
$$

If convergence criterion is satisfied proceed to the next step. Otherwise, go to step 1.

(4) Calculate the force on the solid from the de-coupled method, and obtain the solid moving information [19]. Based on the ALE description, the boundary of the moving body is specified as the velocity of flow equal to that of the body.

- $\quad$ Estimate the solid position (for moving solid case) and apply the ALE to get the mesh velocity and the new flow boundary conditions.

(5) Use the velocity and pressure components in the successive time steps, from the previous time step as initial conditions and the new boundary flow conditions and use the iterative procedure, steps $1-4$. The procedure is repeated until the prescribed time step is reached.

Detailed numerical investigations were conducted to check the convergence characteristics and stability of the numerical scheme developed, for one-dimensional, twodimensional and three-dimensional problems. The model was observed to converge in three to five iterations depending on the problem, time step and mesh size. Various numerical experiments showed that the model is stable for $0 \leq C_{r} \leq 1\left(C_{r}=\right.$ Courant number $\left.=u \Delta t / \Delta x\right)$ and hence a large time step can be used.

The present model with the three-step FEM for the momentum equations was observed to be more stable than other FEM schemes like two-step scheme or Lax-Wendroff schemes due to the third-order accuracy and uniform CFL condition of the scheme. A detailed stability and error analysis for the three-step FEM scheme, used in the present study can be found in Ref. [6].

It should be noted that the solution of the pressure Poisson equation (27) using BEM includes domain integrals while finding out the internal pressure distribution using BEM with a fine mesh, a large system of equations to be solved. This would increase the computational costs, as the system matrices are fully populated. Numerical investigations showed that the computing efficiency could be considerably increased by solving the boundary pressure values on the computational domain using the BEM while the FEM is used to get the internal pressure values.

\section{Model applications}

The presented FEM-BEM model has been applied on three test problems to verify the accuracy and feasibility. The model has been used to simulate initially the cubic cavity flow problem at Reynolds number 100 and has been further used to simulate flow past a fixed square cylinder and finally the movement of a sphere in still fluid.

\subsection{Simulation of cavity flow}

The FEM-BEM model has been applied to verify the numerical procedure. The classical benchmark problem of 'driven flow in a cubic cavity' has been considered for which many numerical model results are available in literature (see Fig. 2). Present model results are compared

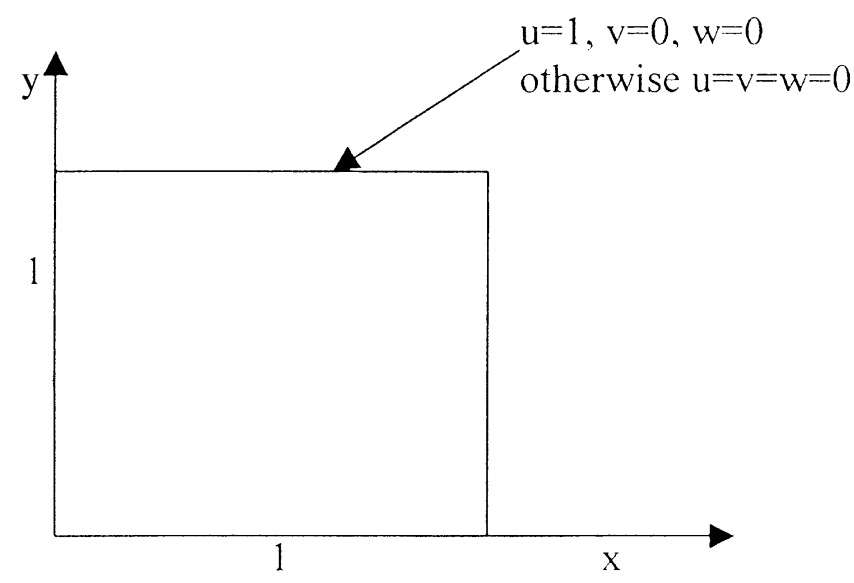

Fig. 2. Cavity flow problem with boundary conditions. 


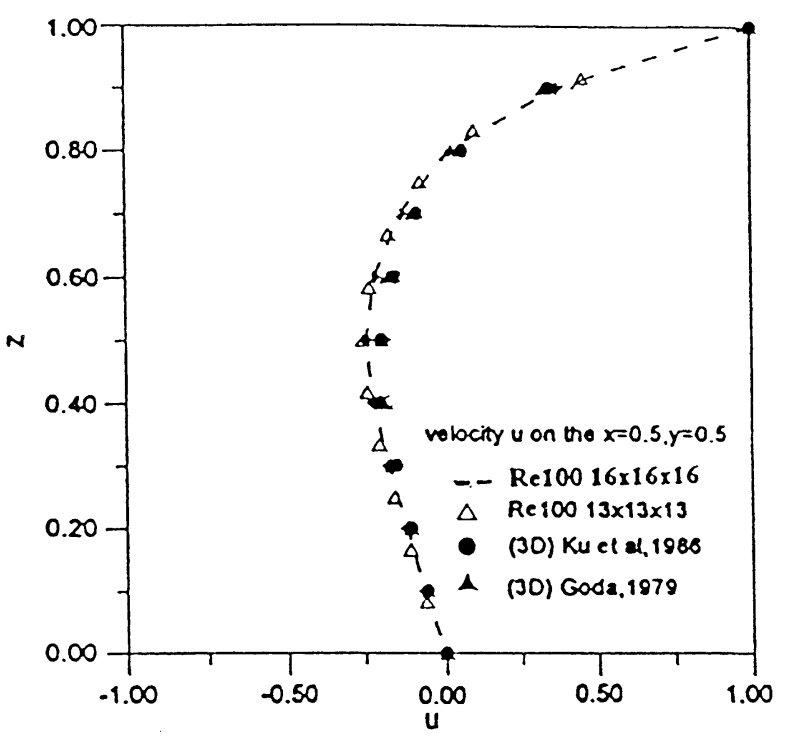

Fig. 3. Velocity profile $(u)$ on vertical centerline of a cubic cavity for $R e=100$.

with the results of Goda [23] and $\mathrm{Ku}$ et al. [24] in threedimensions.

The model problem consists of a cubic cavity with a moving top lid with constant velocity, totally filled with an incompressible viscous fluid. Fig. 2 shows the geometry and boundary conditions of the problem. The present analysis is limited to a computational mesh of $16 \times 16 \times 16$ (on the boundary as well as internal domain).

Here results are presented for Reynolds number $R e=$ 100. Although the computations were performed with a fully explicit scheme in time, only steady state solutions are presented here for brevity. In all the cases, steady states were reached. Fig. 3 shows the $x$-component velocity profiles $(u)$ on the vertical centerline of a cavity

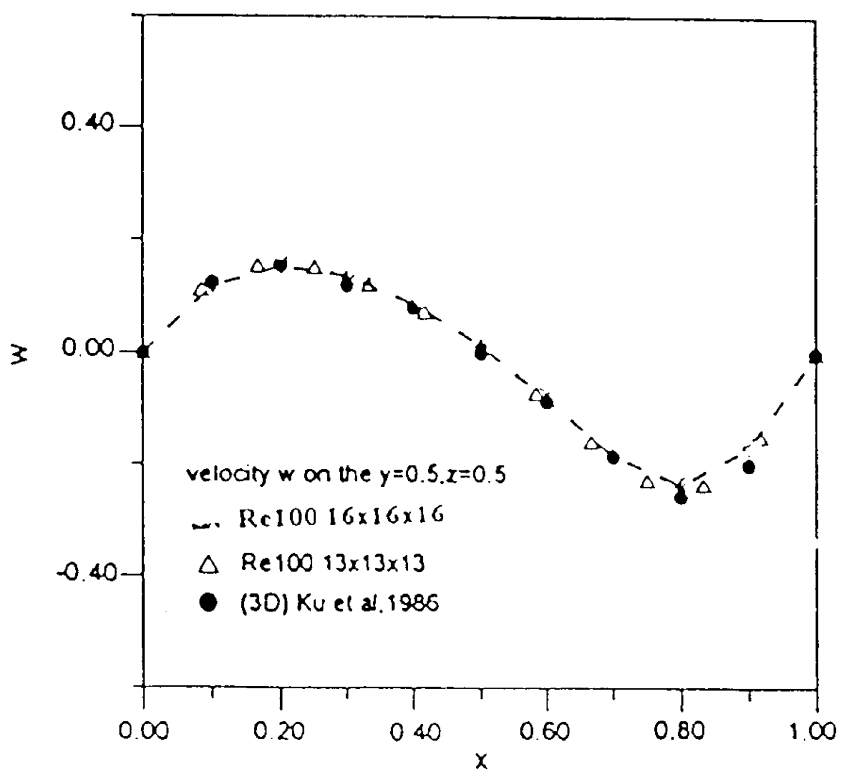

Fig. 4. Velocity profile $(w)$ on horizontal centerline of a cubic cavity for $R e=100$.

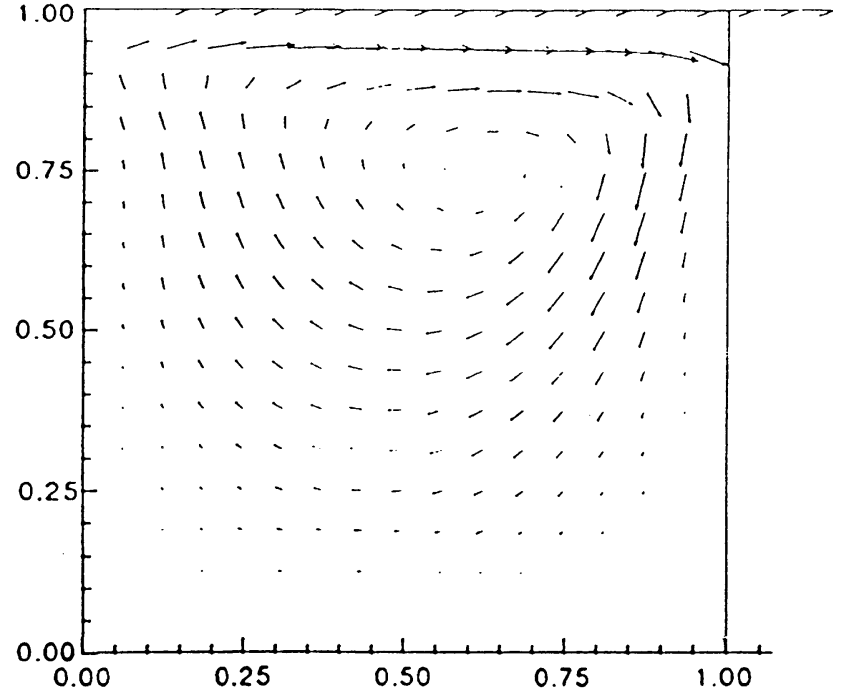

Fig. 5. Profile of the flow vector $(u$ vs $w)$ in the $y=1 / 2$ plane for $R e=100$.

for $R e=100$. Fig. 4 shows the $z$-component velocity profile $(w)$ on the horizontal centerline of the cavity for $R e=100$. The results mostly agree with those by Goda [23] and $\mathrm{Ku}$ et al. [24] considering that the mesh density is coarse. The maximum deviation with the mesh $16 \times 16 \times 16$ is within $5 \%$ in comparison with other model results as can be seen in Figs. 3 and 4. Fig. 5 shows the profile of flow vectors in the $y=1 / 2$ plane for Reynolds number $R e=100$. Its circulation pattern is almost similar to that of two-dimensional cavity flow. All the figures are in reasonably good agreement with the results available in literature and prove the validity of the model in the simulation of three-dimensional incompressible laminar flows.

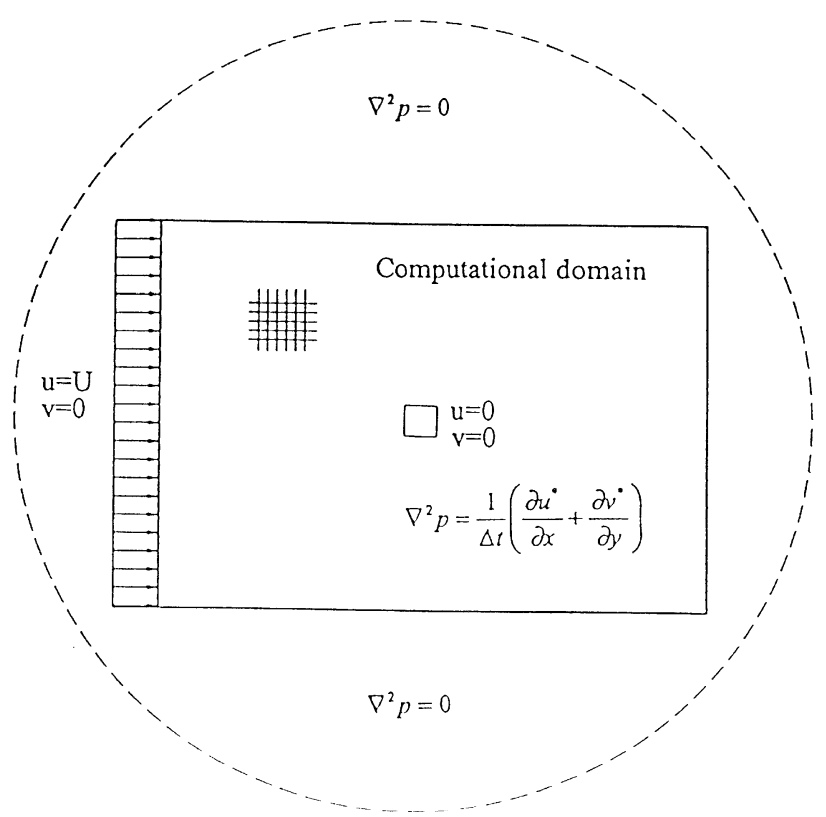

Fig. 6. Computational domain and boundary conditions for 2D problem. 


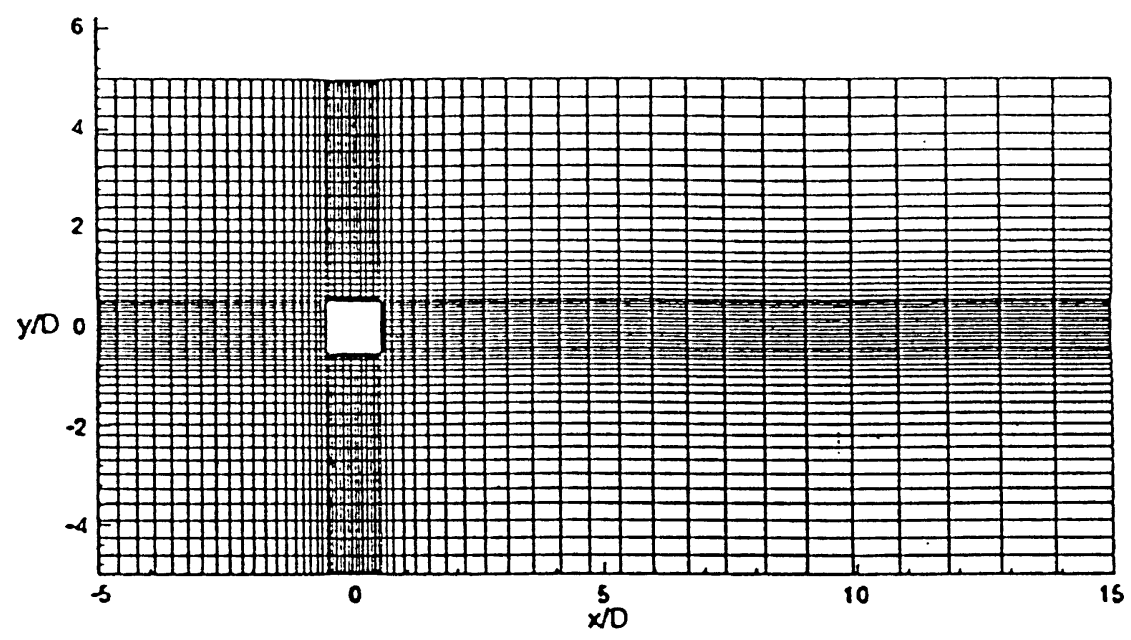

Fig. 7. Mesh for the computational domain for $2 \mathrm{D}$ problem.

\subsection{Flow past a fixed square cylinder}

The flow past a square cylinder phenomenon has been simulated to compare the results with the existing data. A large number of numerical studies have been conducted on the vortex shedding flow, which is produced, by the flow across a fixed square cylinder [25-27]. The present model was tested by simulating flow past a square cylinder for a range of Reynolds numbers of 100, 150, 250 and 500 and compared the results with other studies. Fig. 6 shows the computational domain with boundary conditions. It was 20 units long (cylinder size is unit) and 10 units wide approximately. The discretization of the domain $(65 \times 61)$ using 3440 bilinear elements and 3604 nodes is shown in Fig. 7. The domain of computation was wide enough to encompass the range of the vortex shedding. A time step of 0.05 (non-dimensional) is used in the computation. The boundary conditions were chosen as $u=1, v=0$ at the inlet and a non-slip boundary $u=0, v=0$ on the cylinder surface.

The model was run in unsteady condition and the coefficient of drag and Strauhal numbers were calculated using Eq. (7). Table 1 shows a comparison of the coefficient of drag and Strauhal number at $R e=100,150,250$ and 500 between the results of Davis and Moore [25], Franke et al. [26] and Arnal et al. [27]. Good agreement is observed between the results. Fig. 8 depicts the velocity field and Fig. 9 shows the pressure field for the Reynolds number 500 at time equal to 75 . The vorticity distribution around the cylinder is depicted in Fig. 10. Fig. 11 shows the instantaneous streamlines for the square cylinder for $R e=$ 500. These figures describe the salient features of the flow across the square cylinder problem. As the above figures depict, the numerical simulations with the present model have rendered very reasonable and satisfactory results in comparison with the previous numerical studies.

\subsection{Simulation of the motion of a sphere in still fluid}

The model is applied to simulate the motion of a sphere, with a unit diameter in still infinite fluid of unit density. The sphere is setting into motion impulsively, from the rest to a constant acceleration and finally to a constant velocity. The velocity of the moving sphere is equal to the mesh velocity in the ALE description. Reynolds number is assumed to be 100 for illustration. The computational domain is discretized with 27,920 elements and 29,796 nodes (as in Fig. 12). A cross section of the mesh used is shown in Fig. 13. The acceleration of the sphere (non-dimensional) in the fluid is 100 and a time step of 0.001 is used in the simulation. The total accelerating time is 0.01 .

The time evolution of the drag force with accelerations in the $z$-direction is shown in Fig. 14. The drag force is

Table 1

Comparison of numerical results for the flow across a square cylinder problem

\begin{tabular}{lllll}
\hline Re & Grid & $C_{\mathrm{d}}$ & $S_{t}$ & Author \\
\hline 100 & $41 \times 40$ & 1.64 & 0.147 & Davis and Moore (1982) \\
& $88 \times 76$ & 1.61 & 0.154 & Franke et al. (1990) \\
& $65 \times 61$ & 1.662 & 0.133 & Present \\
& $57 \times 51$ & 1.619 & 0.146 & Present \\
150 & $88 \times 76$ & 1.56 & 0.165 & Franke et al. (1990) \\
& $65 \times 61$ & 1.663 & 0.147 & Present \\
& $57 \times 51$ & 1.607 & 0.159 & Present \\
250 & $41 \times 40$ & 1.76 & 0.161 & Davis and Moore (1982) \\
& $88 \times 76$ & 1.67 & 0.141 & Franke et al. (1990) \\
& $65 \times 61$ & 1.69 & 0.160 & Present \\
& $57 \times 51$ & 1.646 & 0.167 & Present \\
500 & $60 \times 49$ & 1.85 & 0.143 & Arnal et al. (1991) \\
& $40 \times 45$ & 1.80 & 0.173 & Arnal et al. (1991) \\
& $65 \times 61$ & 1.753 & 0.160 & Present \\
& $57 \times 51$ & 1.794 & 0.163 & Present \\
\hline
\end{tabular}




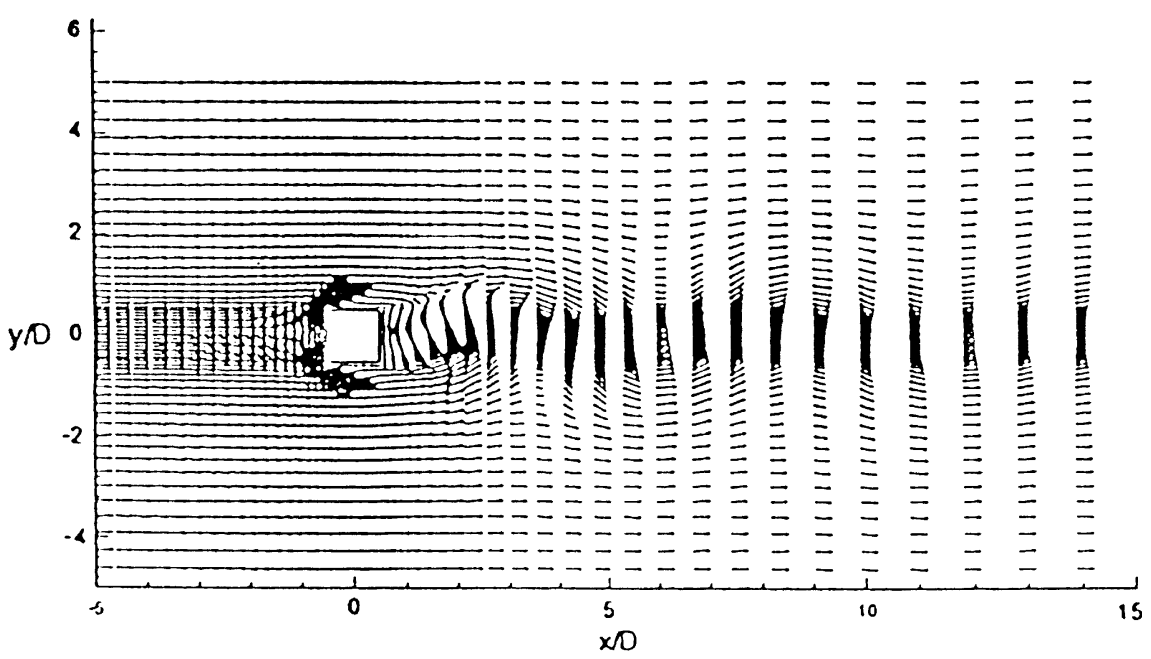

Fig. 8. Velocity field for flow across a square cylinder for $R e=500$ and $t=75$.

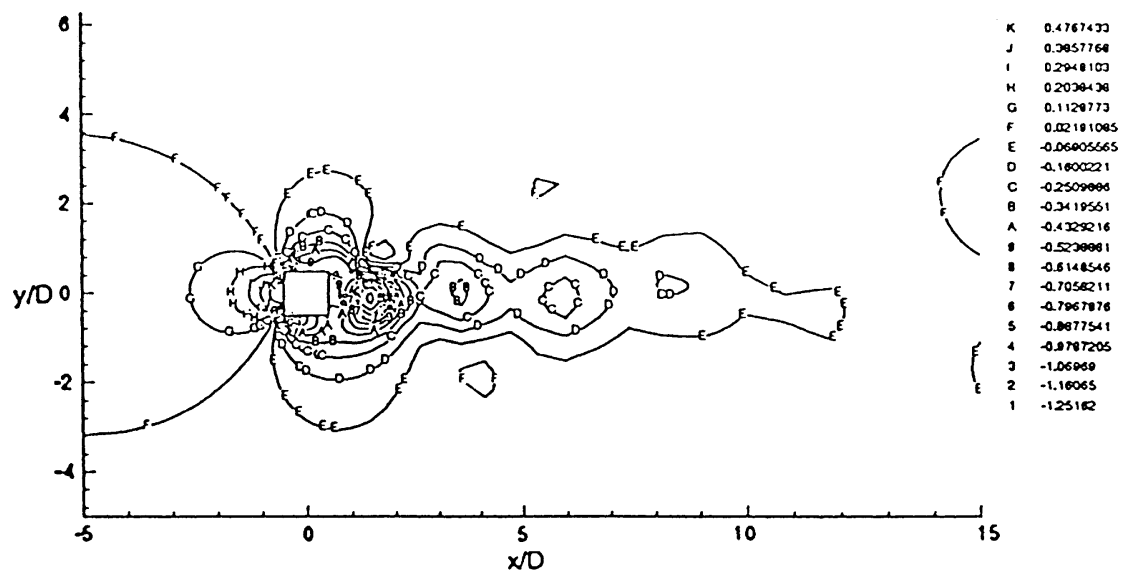

Fig. 9. Pressure field for flow across a square cylinder for $R e=500$ and $t=75$.

dependent on the magnitude of the acceleration. For example, for the case $a=100$, the drag force for the moving sphere in the first time step is equal to 25.98 which is very close to the value 26.18 , that is derived from the added mass concept of the ideal flow theory. This value is reached independently with time interval chosen. Therefore, it can be concluded that for the impulsive motion of an accelerating sphere in a viscous fluid, the drag force

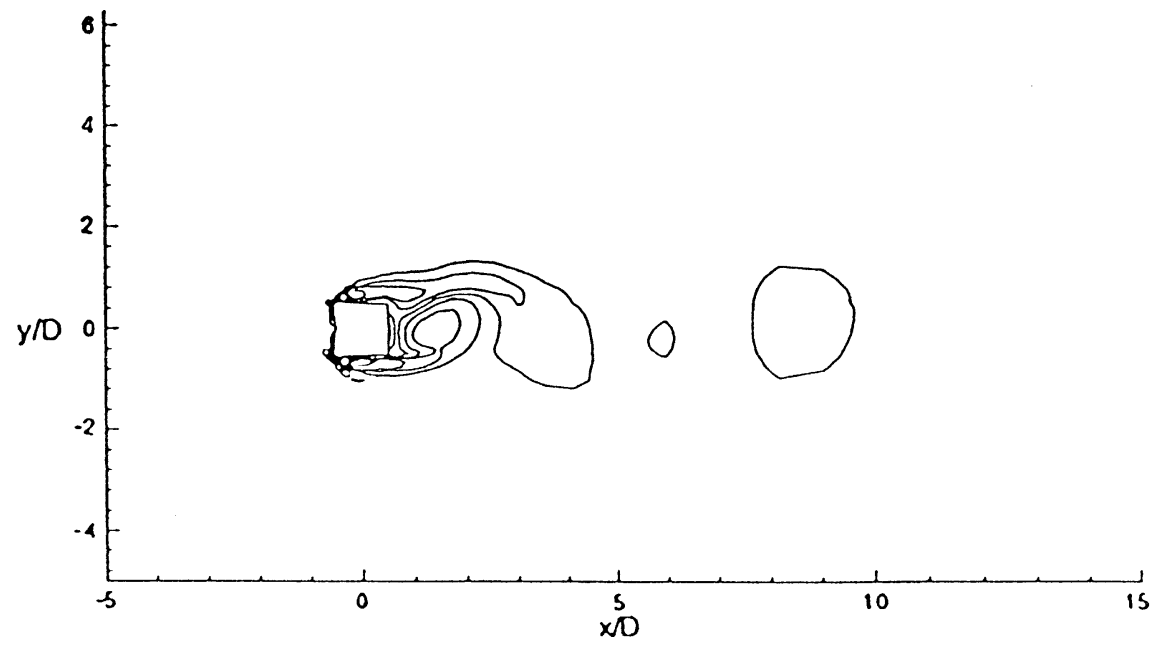

Fig. 10. Vorticity distribution for flow across a square cylinder for $R e=500$ and $t=75$. 


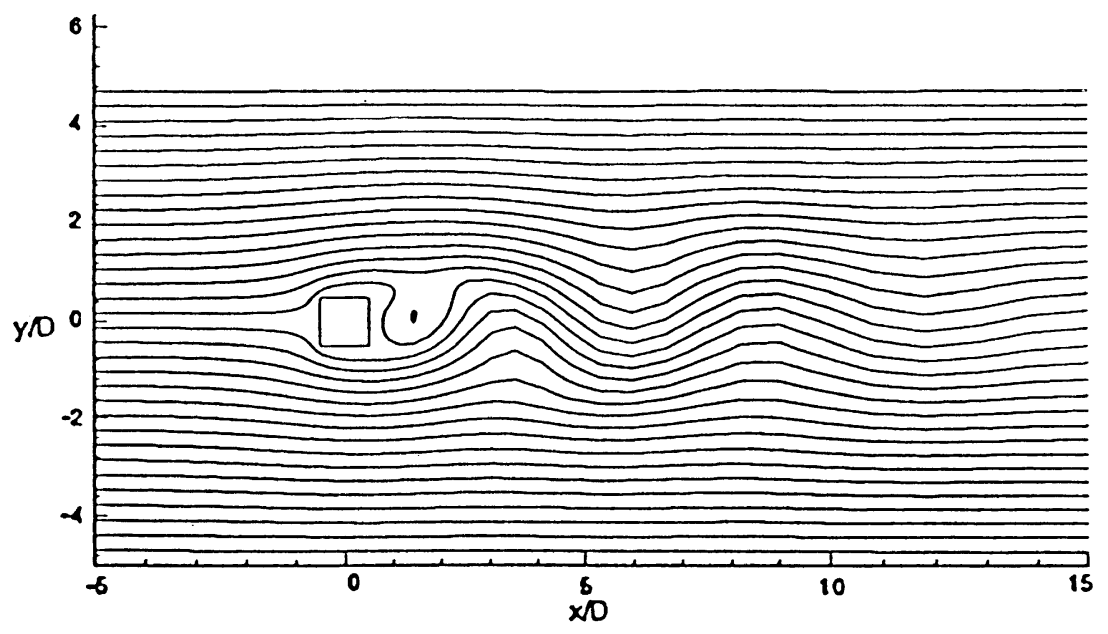

Fig. 11. Instantaneous streamline distribution for flow across a square cylinder for $R e=500$ and $t=75$.

imposed on the sphere can be treated as in the case of an ideal fluid, at the moment of impulsive acceleration. After the first time step of the impulsive acceleration, the drag force increases slowly due to the viscous drag effects, as revealed in Fig. 14.

Figs. 15 and 16 show the velocity field $v$ vs $w$ at $x=1 / 2$ plane and $u$ vs $v$ at $z=1 / 2$ plane, respectively, for the first time step during acceleration. The velocity scale is 0.1 (nondimensional) for the maximum velocity, which occurs at the moving sphere. The velocity distribution in the vertical cross section (at $x=1 / 2$ plane) behaves like a twodimensional circular cylinder moving in a still viscous flow field. The fluid is pushed outside everywhere by the moving sphere. Behind the wake of the sphere, the fluid is refilled by the neighborhood of the moving sphere. However, in the horizontal cross section (at $z=1 / 2$ plane), the velocity field moving outside concentrically, behaves like a source singularity. Figs. 17 and 18 show the

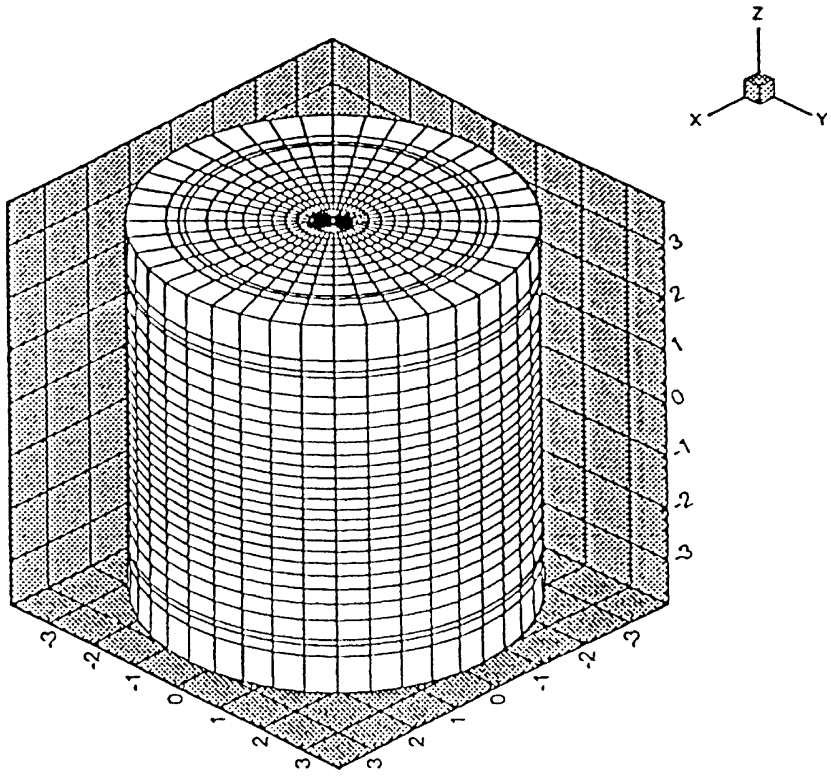

Fig. 12. Domain discretization for the moving sphere in still fluid problem. pressure field at vertical cross section $(x=1 / 2$ plane) and horizontal cross section $(z=1 / 2$ plane), respectively, for the acceleration. At the moment of impulsive acceleration, the pressure distributions at both the vertical and horizontal cross sections show the complete and symmetrical characteristics. After stopping the acceleration impulsively and maintaining at a constant velocity with zero acceleration, the drag force drops immediately, as shown in Fig. 14. The drag force approaches an approximate value of 0.5 (at $t=0.03)$. It is same as predicted by using the drag coefficient as a function of Reynolds number for a smooth sphere from the viscous flow theory which corresponds to an approximate drag coefficient $C_{\mathrm{d}}=1.27$ at $R e=100$ [28].

Figs. 19 and 20 show the velocity field $v$ vs $w$ at $x=1 / 2$ plane and $u$ vs $v$ at $z=1 / 2$ plane, respectively, during deceleration. The velocity scale is 1.0 (non-dimensional) for the maximum velocity, which occurs at the moving sphere. Figs. 21 and 22 show the pressure field at vertical cross

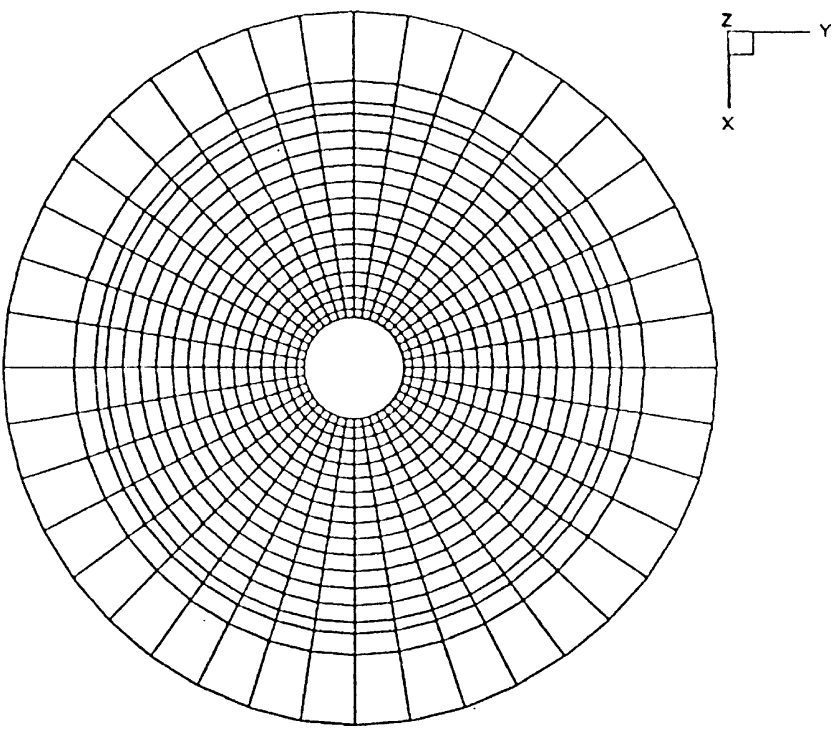

Fig. 13. Cross section of mesh discretization for the moving sphere problem. 


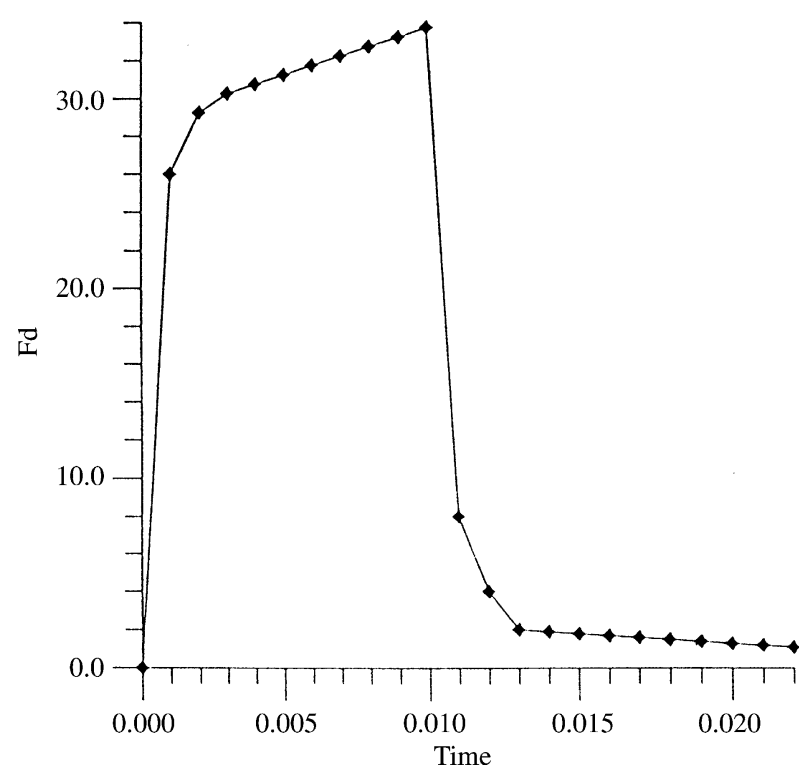

Fig. 14. Time evolution of drag force with acceleration $a=100$ for moving sphere in still fluid problem for $R e=100$.

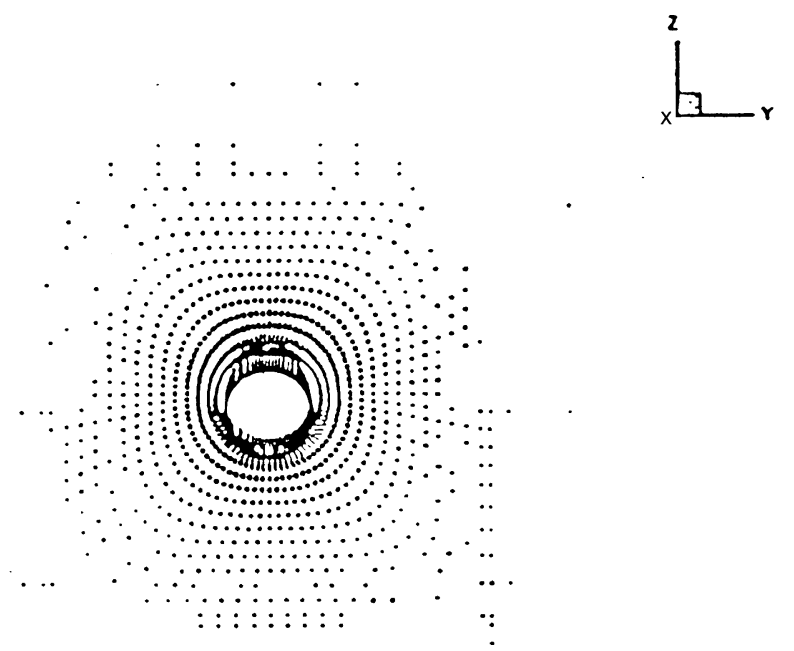

Fig. 15. Local velocity field ( $v$ vs $w$ ) for accelerating sphere problem at $x=1 / 2$ plane for $R e=100$.
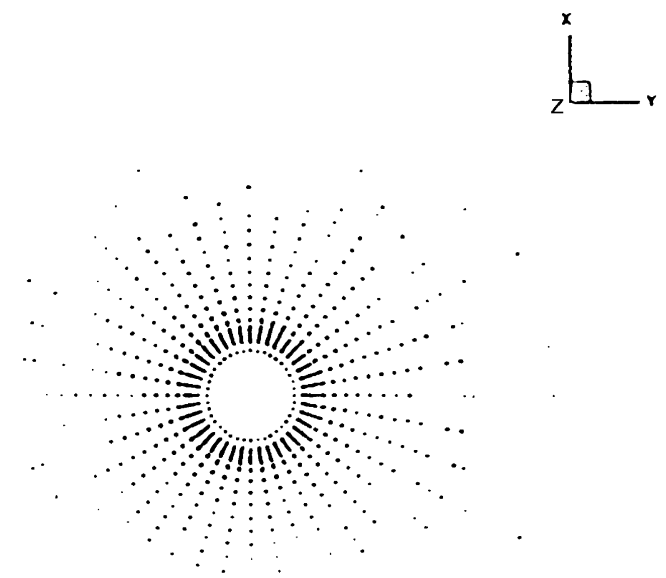

Fig. 16. Local velocity field ( $u$ vs $v$ ) for accelerating sphere problem at $z=1 / 2$ plane for $R e=100$
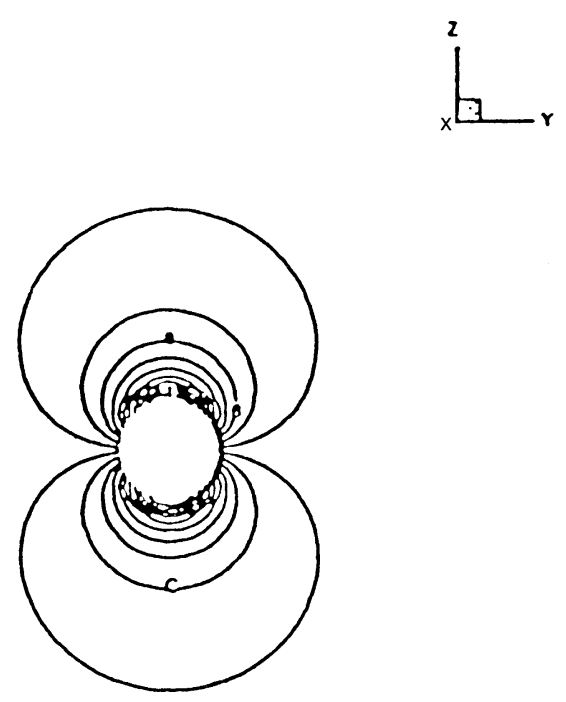

Fig. 17. Pressure distribution for accelerating sphere problem at $x=1 / 2$ plane for $R e=100$.

section $(x=1 / 2$ plane $)$ and horizontal cross section $(z=1 / 2$ plane), respectively, during deceleration. In the deceleration case also, the pressure distribution reveals symmetrical properties in both vertical as well as horizontal cross sections. Concentric pressure distribution is found in the horizontal cross section, showing the property of a source singularity.

Comparing with the drag force due to the added mass, the drag force for a uniformly moving sphere only experiences $1 / 50$ of that of the uniform acceleration of $a=100$. The viscous effect plays only a minor role, as far as contribution to the drag force is concerned. The same characteristics are also observed in the two-dimensional flows such as the motion of a circular cylinder in a still viscous fluid. Young et al. [14] have reported that the drag force for a uniformly moving cylinder expects only $1 / 20$ of that of the uniform acceleration of $a=10$. However, the uniformly

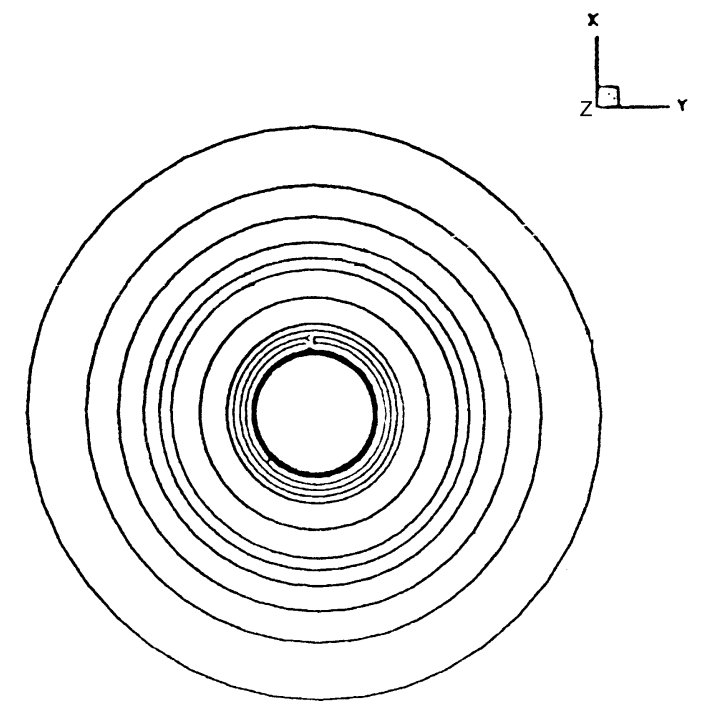

Fig. 18. Pressure distribution for accelerating sphere problem at $z=1 / 2$ plane for $R e=100$. 


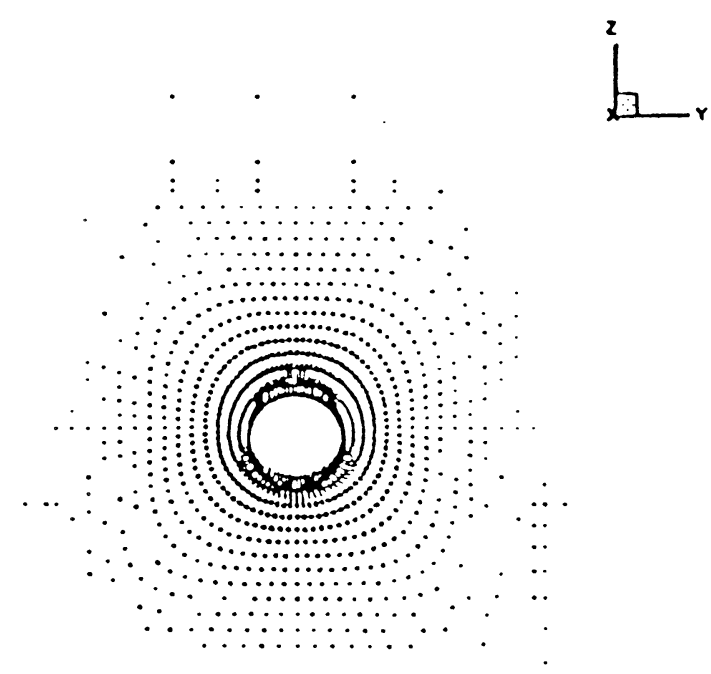

Fig. 19. Local velocity field ( $v$ vs $w$ ) for decelerating sphere problem at $x=1 / 2$ plane for $R e=100$.

moving sphere would experience $1 / 5$ of the drag force as that of the uniform acceleration if the same acceleration $(a=10)$ were used. Under such circumstances, drag force derived by the impulsive acceleration and viscous effect should be taken into consideration. Nevertheless, the present model is able to provide a tool to calculate any state of motion of a sphere in a still viscous flow field. A potential application of the present model is for the analysis of hydrodynamic drag forces on the moving underwater projectiles or the wind structure interaction problems.

\section{Concluding remarks}

A new computational model has been developed to solve three-dimensional incompressible viscous flow problems by the coupling of ALE FEM and BEM. The model based on Navier-Stokes equations in primitive variables is able to
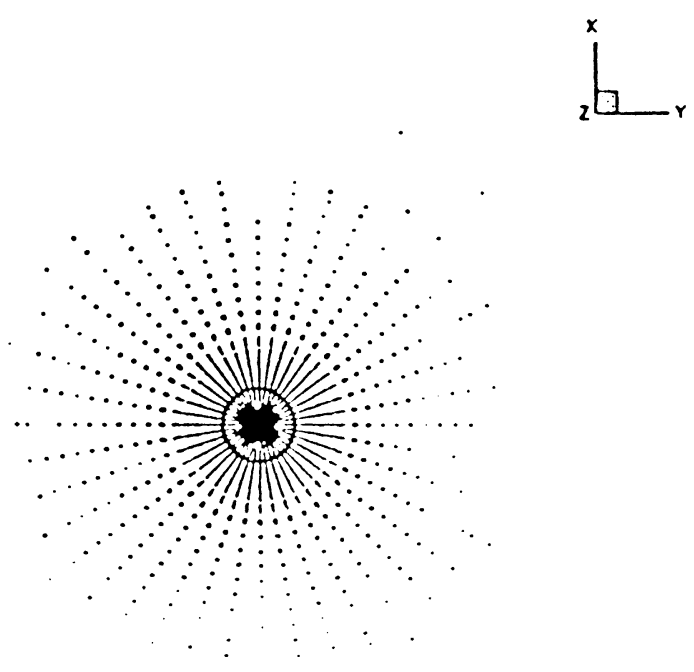

Fig. 20. Local velocity field ( $u$ vs $v$ ) for decelerating sphere problem at $z=1 / 2$ plane for $R e=100$.

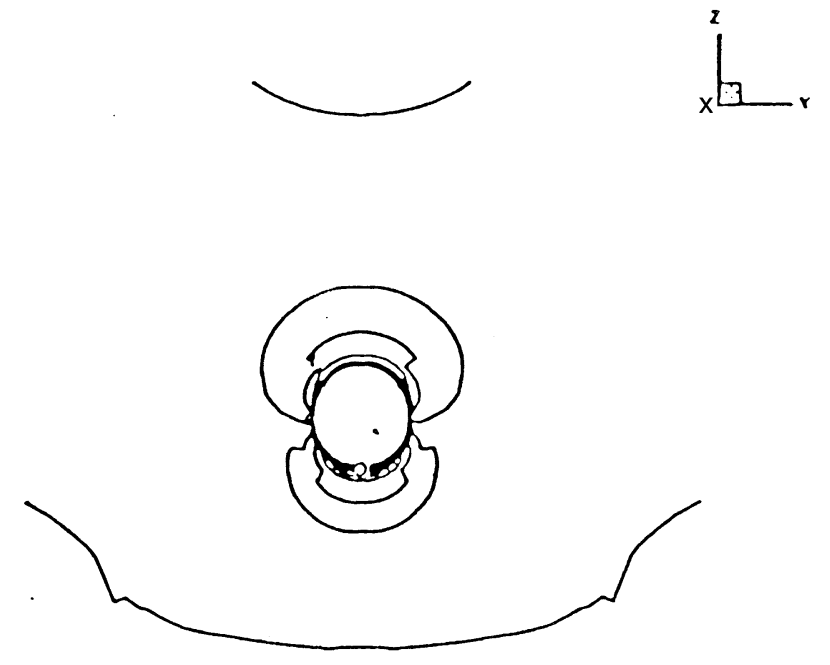

Fig. 21. Pressure distribution for decelerating sphere problem at $x=1 / 2$ plane for $R e=100$.

solve the infinite boundary value problems by extracting the boundary effects on a specified finite computational domain, using the projection method. The Navier-Stokes equations are solved in the model using a three-step FEM and the Poisson type pressure equations are solved using BEM. By coupling FEM and BEM, the model is able to handle infinite domain problems efficiently. The ALE method is used to incorporate the moving boundary problems. The model has been efficiently applied to solve three-dimensional problem of cavity flow, flow past a square cylinder and movement of a sphere in a still fluid. All the applications gave reasonable and satisfactory results and show the feasibility and robustness of the present model. The present model has the potential to compute the hydrodynamic forces on fluidstructure interaction problems with static solid in moving fluid or moving solid in static flow field.
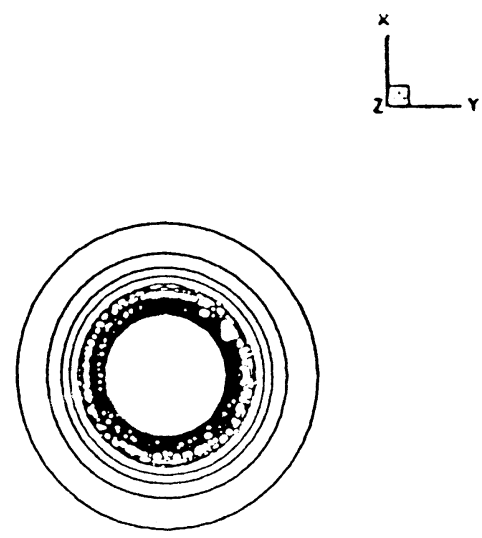

Fig. 22. Pressure distribution for decelerating sphere problem at $z=1 / 2$ plane for $R e=100$. 


\section{Acknowledgements}

The support of the National Science Council, Taiwan, which made this research possible, is deeply appreciated. The constructive comments by reviewers are gratefully acknowledged.

\section{References}

[1] Blevins RD. Flow-induced vibration, 2nd ed. New York: Van Nostrand Reinhold; 1990.

[2] Braza M, Chassaing P, Minh HH. Numerical study and physical analysis of the pressure and velocity fields in the near wake of a circular cylinder. J Fluid Mech 1986;165:79-139.

[3] Licointe Y, Piquet J. On the use of several compact methods for the study of unsteady incompressible viscous flow around a circular cylinder. Comput Fluids 1984;12:255-80.

[4] Brookes AN, Hughes TJR. Streamline upwind/Petrov-Galerkin formulations for convection dominated flows with particular emphasis on the incompressible Navier-Stokes equations. Comput Meth Appl Mech Engng 1982;32:199-259.

[5] Lohner R, Morgan K, Zienkiewics OC. The solution of nonlinear hyperbolic equations systems by the finite element method. Int $\mathrm{J}$ Numer Meth Fluids 1984;4:1043-63.

[6] Jiang CB, Kawahara M. The analysis of unsteady incompressible flows by a three-step finite element method. Int J Numer Meth Fluids 1993;16:793-811.

[7] Power H, Wrobel LC. Boundary integral methods in fluid mechanics. Southampton: Computational Mechanics Publications; 1995.

[8] Belytschko T, Kennedy JM. Finite element approach to pressure wave attenuation by reactor fuel subassemblies. J Press Technol 1975; 172-7.

[9] Huerta A, Liu WK. Viscous flow with large free surface motion. Comput Meth Appl Mech Engng 1988;69:77-324.

[10] Noh WF. CEL: a time-dependent two-space-dimensional coupled Eulerian-Lagrangian code. In: Alder BJ, Fernbach S, Rotenburg M, editors. Methods in computational physics, vol. 3. New York: Academic Press; 1964

[11] Hirt CW, Amsden AA, Cook JL. An arbitrary Lagrangian-Eulerian computing method for all flow speeds. J Comput Phys 1974;14:227-53.

[12] Donea J, Fasoli-stella P, Giuliani S. Lagrangian and Eulerian finite element technique for transient fluid structure interaction problems.
Transactions of the Fourth International Conference on Structural Mechanics in Reactor Technology, Paper B1/2; 1977.

[13] Hughes TJR, Liu WK, Zimmermann TK. Lagrangian-Eulerian finite element formulation for incompressible viscous flows. Comput Meth Appl Mech Engng 1981;29:329-49.

[14] Young DL, Chang JT, Eldho TI. A coupled BEM and arbitrary Lagrangian-Eulerian FEM model for the solution of two-dimensional laminar flows in external flow fields. Int J Numer Meth Engng 2001; 51(9):1053-7.

[15] Young DL, Huang JL, Eldho TI. Simulation of laminar vortex shedding flow past cylinders using a coupled BEM and FEM model. Comput Meth Appl Mech Engng 2001;190:5975-98.

[16] Young DL, Huang JL, Eldho TI. Numerical simulation of highReynolds number flow around circular cylinders by a three-step FEM-BEM model. Int J Numer Meth Fluids 2001;37:657-89.

[17] Liggett JA. Fluid mechanics. New York: McGraw-Hill; 1994.

[18] Debler WA. Fluid mechanics fundamentals. Englewood Cliffs, NJ: Prentice-Hall; 1990.

[19] Nomura T, Hughes TJR. An arbitrary Lagrangian-Eulerian finite element method for interaction of fluid and rigid body. Comput Meth Appl Mech Engng 1992;95:115-38.

[20] Young DL, Liao CB, Sheen HJ. Computations of recirculation zones of a confined annular swirling flow. Int J Numer Meth Fluids 1999;29: $791-810$.

[21] Reddy JN. An introduction to the finite element method. New York: McGraw-Hill; 1993.

[22] Brebbia CA, Telles JCF, Wrobel LC. Boundary element techniquestheory and applications in engineering. Berlin: Springer; 1984.

[23] Goda K. A multistep technique with implicit difference schemes for calculating two- or three-dimensional cavity flows. J Comput Phys 1979;30:76-95.

[24] Ku HC, Hirsh RS, Taylor TD. A pseudo-spectral method for solution of the three-dimensional incompressible Navier-Stokes equations. J Comput Phys 1986;70:439-62.

[25] Davis RW, Moore EF. A numerical study of vortex shedding from rectangles. J Fluid Mech 1982;116:475-506

[26] Franke R, Rodi W, Schonung B. Numerical calculation of laminar vortex shedding flow past cylinders. J Wind Engng Ind Aerodyn 1990; 35:237-57.

[27] Arnal MP, Goering DJ, Humphrey JAC. Vortex shedding from a bluff body adjacent to a plane sliding wall. J Fluids Engng 1991;113: 384-98.

[28] Schlichting H. Boundary-layer theory, 6th ed. New York: McGrawHill; 1968 